\title{
QUEEN'S
UNIVERSITY
BELFAST
}

\section{Irradiation and dosimetry arrangement for a radiobiological experiment employing laser-accelerated protons}

Polin, K., Doria, D., Romagnani, L., Chaudhary, P., Cirrone, G. A. P., Maiorino, C., Milluzzo, G., Petringa, G., Romano, F., Schettino, G., Scuderi, V., Prise, K., \& Borghesi, M. (2019). Irradiation and dosimetry arrangement for a radiobiological experiment employing laser-accelerated protons. Journal of Instrumentation, 14(10), [C10015]. https://doi.org/10.1088/1748-0221/14/10/C10015, https://doi.org/10.1088/1748-0221/14/10/C10015

Published in:

Journal of Instrumentation

Document Version:

Peer reviewed version

Queen's University Belfast - Research Portal:

Link to publication record in Queen's University Belfast Research Portal

Publisher rights

Copyright 2019 IOP. This work is made available online in accordance with the publisher's policies. Please refer to any applicable terms of use of the publisher.

\section{General rights}

Copyright for the publications made accessible via the Queen's University Belfast Research Portal is retained by the author(s) and / or other copyright owners and it is a condition of accessing these publications that users recognise and abide by the legal requirements associated with these rights.

Take down policy

The Research Portal is Queen's institutional repository that provides access to Queen's research output. Every effort has been made to ensure that content in the Research Portal does not infringe any person's rights, or applicable UK laws. If you discover content in the Research Portal that you believe breaches copyright or violates any law, please contact openaccess@qub.ac.uk. 


\title{
Irradiation and Dosimetry Arrangement for a Radiobiological Experiment Employing Laser- Accelerated Protons
}

\author{
K. Polin ${ }^{1}$, D. Doria ${ }^{1,2}$, L. Romagnani ${ }^{1,3}$, P. Chaudhary ${ }^{1}$, G.A.P. Cirrone ${ }^{4,5}$ C. \\ Maiorino ${ }^{1}$, G. Milluzzo ${ }^{1,4}$, G. Petringa ${ }^{4}$, F. Romano ${ }^{6,4}$, G. Schettino ${ }^{6}$, V. Scuderi ${ }^{5,4}$, \\ K. Prise ${ }^{1}$ and M. Borghesi ${ }^{1}$ \\ 1. Queen's University Belfast, \\ University Road, Belfast, United Kingdom \\ 2. ELI-NP, \\ Strada Reactorului, Magurele, Romania \\ 3. LULI-École Polytechnique, \\ École Polytechnique, Palaiseau cedex, France \\ 4. INFN-LNS, \\ Via Santa Sofia, 62, Catania, Italy \\ 5. Institute of Physics ASCR, v.v.i. (FZU), ELI-Beamlines Project, Prague, Czech Republic \\ 6. National Physical Laboratory, \\ Hampton Road, Teddington, Middlesex, United Kingdom \\ E-mail: kpolin01@qub.ac.uk
}

ABSTRACT: The Bragg Peak is a unique characteristic of ion beams which makes their use beneficial in cancer treatment as it allows the majority of dose deposition to be localised in a precise volume. The use of a laser system for particle acceleration is currently investigated as a potential alternative to the conventional RF accelerators currently used for hadrontherapy. The biological response of cells irradiated by high dose-rate laser-driven ions is currently being explored in this context. The experiment reported here was carried out as part of the A-SAIL project, a UK-wide collaboration aiming to develop innovative techniques for accelerating ions for future clinical applications. In this contest, a radiobiological experiment was carried out on the pico2000 laser beamline at LULI-École Polytechnique using an energetically spread, laseraccelerated proton beam. This paper will discuss the arrangement used in the experiment, and the techniques employed for an accurate estimation of the dose deposited, supported by Monte Carlo simulations of the particle propagation through the irradiation system.

KEYWORDS: Lasers; Ion Sources; Radiation damage evaluation methods. 


\section{Contents}

1. Introduction 2

2. Irradiation Source Arrangement: LULI2000 Laser 2

3. Cell Irradiation Experimental Set-Up 3

4. Determination of Beam Energy and Absorbed Dose 4

\section{Introduction}

Ions and protons have shown many advantages for cancer treatment via radiotherapy due to their characteristic inverse dose-depth deposition profile (Bragg Peak), allowing a controlled dose deposition within the tumour region with a higher ballistic precision than X- and gamma rays, and minimizing the damage to healthy tissues surrounding the tumour. Large-scale RF machines such as cyclotrons, synchrotrons or linacs are used to accelerate protons and light ions for hadrontherapy, which is still a costly and not widely available form of treatment. There is interest in exploring a laser-based approach as an alternative method of particle delivery, which may enhance in the future the availability and effectiveness of particle therapy.[1] Nevertheless due to the peculiarities of laser-accelerated particles such as the ultra-short bunch duration (order of ns) and, consequently, the extremely high dose rate $\left(\geq 10^{9} \mathrm{~Gy} / \mathrm{s}\right.$ [2]), further studies need to be carried out to demonstrate the suitability of such beams for medical as well as radiobiological applications. The A-SAIL project aims to develop laser-driven acceleration to achieve ion beams as well as new technologies for cell irradiation and future clinical applications. In particular, a thorough assessment of the radiobiological effectiveness of such high-dose rate beams on cells is crucial in order to predict lethal and sub-lethal effects caused by these highly pulsed beams.

In this framework, a recent experiment aiming at studying the radiobiological effectiveness of laserdriven protons on biological samples was carried out at the LULI Laboratory and will be described in this paper, focusing in particular on the irradiation and dosimetry setup employed. The accurate estimation of the dose delivered to the cells in such an unconventional environment, by using for instance dose-rate independent dosimeters sensitive to high flux and dose, is extremely important for an accurate estimation of the dose effect on DNA damage in such extreme conditions.

\section{Irradiation Source Arrangement: LULI2000 Laser}

The experiment was carried out using the pico2000 beamline of the LULI2000 laser at LULI-École Polytechnique. The laser beamline delivers $80 \mathrm{~J}$ in $\approx 1$ ps pulses at $1 \omega(\lambda=1.054 \mu \mathrm{m})$ at a repetition rate of approximately 1 shot every 90 minutes. A f=800 mm, f/4 off-axis parabola was used to focus the pulse at an angle of $\sim 22.5^{\circ}$ upon gold foil targets of $25-50 \mu \mathrm{m}$ thickness from which contaminant protons were accelerated through the Target-Normal Sheath Acceleration (TNSA) mechanism. The irradiation set-up was tailored to isolate and utilize protons of approximately $10 \mathrm{MeV}$ from the characteristically multienergetic TNSA spectrum. 
The protons were energetically dispersed via a $1 \mathrm{~T} 10 \mathrm{~cm}$ magnetic dipole coupled with an $800 \mu \mathrm{m}$ pinhole before exiting the interaction chamber through a $50 \mu \mathrm{m}$ Kapton window to be directed onto the cells, placed within a re-entrance tube. The dose delivered to cells was to be measured through using two types of passive dose-rate independent dosimeters (CR-39 and Radiochromic Film, or RCF) which will be described in the following section. This setup can be seen in Figure 1.

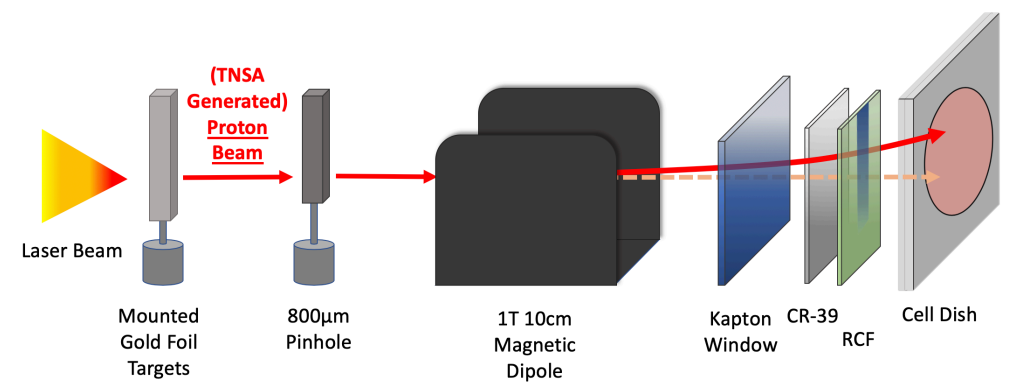

Figure 1. Diagram of the setup inside the pico2000 interaction chamber.

\section{Dosimetry and Cell Irradiation Experimental Setup}

Two dosimeters were used in this experiment to allow the measurement of the dose delivered to the cells; Gafchromic EBT3 films (customised by the absence of the standard protective topcoat layer, which would have impeded low-energy protons from reaching the cells) and Columbia Resin \#39 (CR39 , a plastic polymer used as a solid-state nuclear track detector to measure particle radiation). These dosimeters provide information on the beam and energy spread, independent from one another at a shotby-shot basis; EBT3 and CR-39 allow the dose, dose uniformity and particle fluence to be measured, and can be correlated with each other to gain a full understanding of the dosimetry within the experiment. EBT3 films are a type of RCF which are self-developing under the application of ionising energy, and are sensitive in the $0.1-10$ Gy region. They are scanned and analysed post-irradiation to gain information on the optical density (OD) of the RCF's active layer. The OD is then converted to dose using a previously determined dose calibration, obtained in the LNS-INFN facility in Catania, Italy, using $60 \mathrm{MeV}$ protons accelerated by a Tandem accelerator. Although the proton particles utilised in this experiment were of a much lower energy $(\sim 10 \mathrm{MeV})$, no significant variation in the film's response is expected at this energy [3], and thus the calibration can be applied without the need for correction factors.

The positioning of the setup of these dosimeters can be seen in Figure 1. The customised EBT3 (labelled 'RCF' in the diagram) was placed immediately in front of the cell dish so that the exposed active layer directly faced the cell dishes' $3 \mu$ m mylar window rather than the beam; this ensured that there was minimal dose difference between the RCF and cells. The Monte Carlo simulation code Geant4 [4] was utilised to confirm if this difference in dose was significant, and they determined that there was a $0.2 \%$ energy loss between the RCF's active layer and the seeded cells for $9.5 \mathrm{MeV}$ protons. Therefore, the corresponding dose difference between the two surfaces is considered negligible.

Multiple types of biological assays were carried out for both HUVEC (human umbilical endothelial vein cells) and AGO1522 (human fibroblast cells); Clonogenic [5], Senescence [6], and Immunofluorescence (DNA damage) assays [7]. The cells were grown on mylar secured by stainless steel dishes, customised so that the cell medium can be injected and extracted as needed. Cells were either seeded across the whole mylar layer (for the immunofluorescence assay), or spot-seeded at a precise location. The cell dishes were placed within the re-entrance pipe, a few centimetres from the 
Kapton window; this small distance of air ensured that the amount of scattering was minimized, therefore minimizing energy loss before the ion pulse reached the radiochromic films (RCF) and cells.

\section{Determination of Beam Parameters and Absorbed Dose}

The energy spectrum of the proton beam was measured using Radiochromic Film (RCF) stacks. These stacks comprise of layers of HD-V2, a separate type of RCF which is much less dose-sensitive than EBT3 (with the capacity to distinguish doses between $10-1000 \mathrm{~Gy}$, as opposed to approximately 0.25 - 10 Gy, respectively.) This lessened sensitivity means that the stacks can be placed directly in the beamline, before the magnetic dipole, in order to provide information of the energies within the beam pulse; higher energy protons will travel further through the RCF stack than lower energy protons. These stacks showed energies ranging from a few $\mathrm{MeV}$ up to approximately $20 \mathrm{MeV}$.

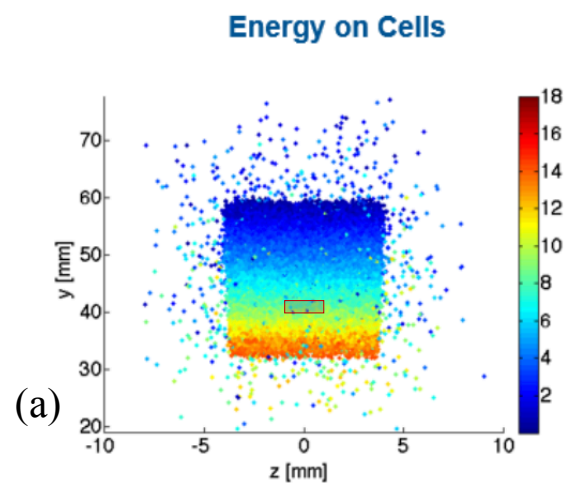

(b)
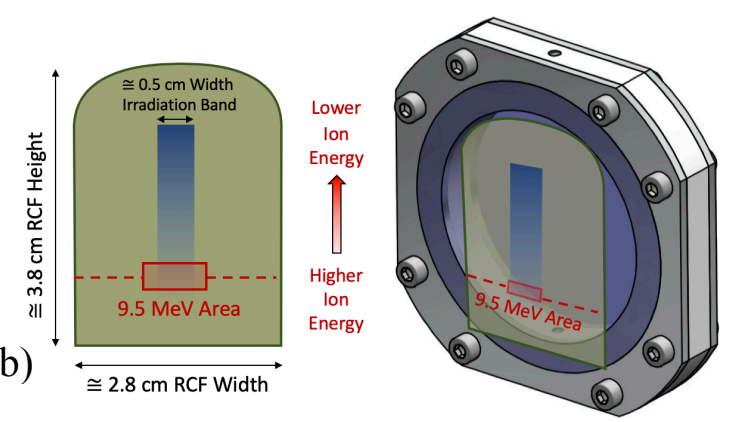

Figure 2. (a) Monte Carlo simulation showing the proton energy spread at the position of the cells. The y-axis indicates the distance from the zero point, and the coloured legend denotes the energy spread in $\mathrm{MeV}$. The box indicates the energetic area of interest; the $\approx 9.5 \mathrm{MeV}$ energy region.

(b) An example irradiated $R C F$, and a schematic of the cell dish with the RCF placed in front of the mylar window where the cells are grown. The horizontal line shows the $9.5 \mathrm{MeV}$ Proton position where the dose measurement was taken from, and the red box indicates the area where the cells were seeded.

Due to the particles passing through a magnetic dipole, the proton pulses were dispersed in accordance to their energies. In order to predict the energy dispersion on the cell plane, the experimental setup was modelled using Geant4 [6], as shown in Figure 2a, which shows the energy spread of the dispersed proton beam in the vertical direction from the base of the RCF. We chose to work at $\sim 9.5 \mathrm{MeV}$, where simulations predicted delivery of a 1 Gy dose. A $3 \times 4 \mathrm{~mm}^{2}$ cellular spot was centred upon the $9.5 \mathrm{MeV}$ proton line with a $\Delta \mathrm{E} / \mathrm{E}$ of about $5 \%$. In such configuration, the proton bunch reached the cells with a time duration of about $\approx 400 \mathrm{ps}$ and a dose rate of $\sim 2.5 \times 10^{9} \mathrm{~Gy} / \mathrm{s}$ (assuming 1 Gy delivered per pulse). Once the vertical position of the cells corresponding to the energy of interest was determined, the dose profiles over the selected region can be extracted from the analysis of the 2D dose images obtained with the RCF placed in front of the cell plane. Figure $2 b$ shows the RCF image acquired with a commercial scanner EPSON V750 Pro scanner, for it to then be processed with ImageJ [8], providing the conversion from pixel values to optical density for the specific scanner use. 
An optical density-dose calibration of the customized EBT3, (obtained through using RF accelerated protons at INFN-LNS) was then applied to retrieve the 2D dose distribution and profiles. Fig 3 shows the transverse dose profile at $9.5 \mathrm{MeV}$ obtained for a representative shot. As one can see, a flat profile is measured allowing a uniform dose irradiation on the cells along the transverse direction; in this case, an average dose of 1.02 Gy with a standard deviation of $0.01 \mathrm{~Gy}$ is obtained in the region under observation.

A total uncertainty on the dose delivered per shot of less

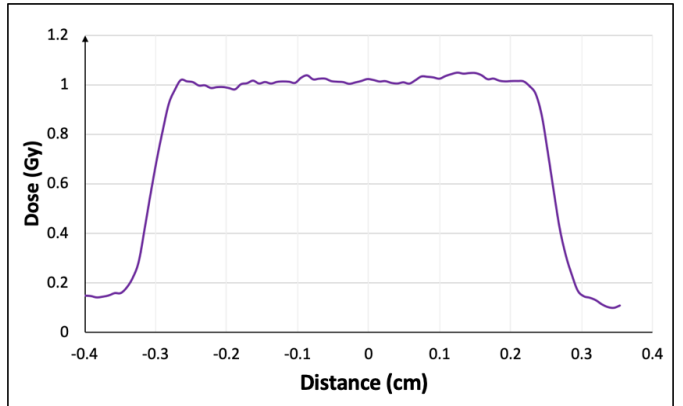

Figure 3. An example dose measurement across the $9.5 \mathrm{MeV}$ line on an $\mathrm{RCF}$, showing an average dose of $1.0 \pm 1 \% G y$. than $5 \%$ has been estimated considering the error on the RCF calibration and the uncertainty on the OD measurement.

A shot-to-shot fluctuation in the dose delivered was observed under the same nominal experimental conditions, which was of the order of $12 \%$. This error was determined through first evaluating the doses and standard deviations across 26 separate shots, and then calculating the percentage difference of the average standard deviation to average dose. This is a reasonable stability for performing radiobiology experiment, particularly when coupled to on-shot dosimetry using the method described above.

This experimental set-up was successfully employed for a number of radiobiological assays which will be reported in a separate publication.

\section{References}

[1] K.W. Ledingham et al, Towards laser driven hadron cancer radiotherapy: a review of progress. Appl. Sci. 4, 402-443 (2014),

[2] Friedl A.A, Schmid. T. E. Application of Laser-Driven Beams for Radiobiological Experiments, in Applications of Laser-Driven Particle Acceleration (CRC, 2018)

[3] S. Reinhardt et al, Investigation of EBT2 and EBT3 films for proton dosimetry in the 4-20 MeV energy range, Radiat Environ Biophys, 54:71-79 (2015)

[4] Geant4; A Simulation Toolkit. 2019. Overview. [ONLINE] Available at: https://geant4.web.cern.ch/. [Accessed 6 February 2019]

[5] N.A.P. Franken et al., Clonogenic assay of cells in vitro, Nature Protocols, vol. 1, pp 2315-2319 (Dec 2006).

[6] K.K.C. Tsai et al, Low-dose radiation-induced senescent stromal fibroblasts render nearby breast cancer cells radioresistant, Radiation Research, vol. 172, pp 306-313 (2009).

[7] P. Chaudhary et al, Relative biological effectiveness variation along monoenergetic and modulated bragg peaks of a 62-mev therapeutic proton beam: A preclinical assessment, International Journal of Radiation Oncology Biology Physics, vol. 90, no. 1, pp 27-35 (2014).

[8] ImageJ: Imaging Processing and Analysis in Java. 2019. [ONLINE] Available at: https://imagej.nih.gov/ij/. [Accessed 7 February 2019] 